\title{
USER-ADAPTIVE NOTIFICATION SOUND ASSIGNMENT FOR NOTIFICATION MESSAGES
}

\author{
Marina Sakai ${ }^{1}$, Junko Shirogane ${ }^{2}$, Hajime Iwata ${ }^{3}$ and Yoshiaki Fukazawa ${ }^{1}$ \\ ${ }^{1}$ Waseda University, Fukazawa Lab., Building 63, Waseda University, 3-4-1 Ohkubo, Shinjuku-ku, Tokyo 169-8555, \\ Japan \\ ${ }^{2}$ Tokyo Woman's Christian University, 2-6-1 Zempukuji, Suginami-ku, Tokyo 167-8585, Japan \\ ${ }^{3}$ Kanagawa Institute of Technology, 1030 Shimo-Ogino Atsugi Kanagawa 243-0292, Japan
}

\begin{abstract}
When using software, various notification messages are displayed in response to users' operations. Although each notification message has its own importance level, users often do not perceive the importance level. For example, they just proceed with an operation without reading the content or they do not notice the notification itself. Meanwhile, sound is a common resource used to convey the importance of a situation and is often employed to make users aware of notification messages. Thus, it is effective to represent the importance of the notification message by sound. However, sound importance depends on the user. This paper proposes a determination method of notification sounds by evaluating the importance level of sounds by users. Then sounds are assigned to notification messages according to users' input.
\end{abstract}

\section{KEYWORDS}

Notification Messages, Notification Sound, User-Adaptive, Assignment

\section{INTRODUCTION}

Notification messages are displayed as users interact with software to indicate what the user has done, to represent a problem, or to convey information. There are various kinds of notification messages, and each one has a different level of importance. However, there are many cases in which users do not properly understand the importance of a notification message. For example, users often proceed with an operation without reading the content of the message or they completely miss a notification message. If users execute an operation without recognizing the intent of the notification message, important operations, which cannot be undone such as deleting files or paying money may be unconsciously performed.

Meanwhile, sound is a common resource used to convey the importance of a situation. Examples include sounds of an ambulance siren and an earthquake early warning. Sound can be recognized even when users do not pay attention to the situation. In addition, a notification sound can alert users to a notification message. Thus, it is effective to represent the importance of the notification message by sound. However, the feeling of a sound depends on the user. For example, when some people hear a scratching sound on a blackboard, they want to run away immediately, while others feel it is unpleasant but bearable. Thus, what sounds sound important depends on the user's perception.

In this study, we propose a method to assign notification sounds to notification messages according to individual user's senses so that users notice and respond appropriately to important notification messages. In a previous study (Sakai et al., 2019), we assumed that users paid attention to unpleasant sounds. Thus, we proposed a method to assign more unpleasant sounds to more important notification messages. The results of user experiments based on the proposed method showed that the assumption was appropriate. If appropriate sounds can be assigned, they can be useful for notification messages according to individual user's feelings. However, the feedback contained many comments about the assigned sound such as "I noticed the sound, but the sound is too strong for the intent of the notification message" or "The sound startled me", and "the evaluation of the sound assigned to the notification message was exaggerated compared to the message". Thus, we concluded that unpleasant sounds are inappropriate for users to notice important notification messages. In this study, we survey characteristics of sounds to inform of important messages in an effort to improve our method to evaluate and assign sounds to notification messages. This study should help users recognize the importance of notification messages according to their feelings, even if the notice is not read. 


\section{BACKGROUND}

\subsection{Earthquake Early Warning}

According to the Japan Meteorological Agency (2007), the Earthquake Early Warning (EEW) System is designed to predict the arrival time and intensity of strong earthquakes in various areas and then inform the public as quickly as possible before strong earthquakes arrive. It is intended to protect people, slow down trains, and control machinery in factories. In other words, an earthquake early warning can be considered a high-importance notification that alerts people to danger and allows them to respond immediately and appropriately. The earthquake early warning is accompanied by a sound. Ifukube (2013), the creator of the earthquake early warning sound, mentioned the following points when creating the sound: (1) the sound conveys urgency, (2) it does not give a sense of uncomfortability or anxiety, (3) it is audible even in noisy environments, (4) it is audible to people with mild hearing impairment, and (5) it is not similar to a sound heard somewhere else.

Since this study aims to enable users to recognize important notification messages and take appropriate actions, we believe that the emergency earthquake early warning is an appropriate reference for high-importance notifications.

\subsection{Notification Sound}

The macOS Human Interface Guidelines (2021) mention that sound is a great way to get users' attention when they are not viewing the screen. Thus, the guidelines instruct developers to provide sound to supplement notification messages. As an example, the To-Do list app plays a notification sound to inform of the time to perform an important task. The guidelines mention that apps can use custom or built-in sounds, but if developers use custom sounds, they should ensure that the sounds are short, distinctive, and professional.

Microsoft Windows Dev Center (2020) mentions that sound is the audio element of the user experience, and when used properly, can be an effective form of communication that establishes a non-verbal and even emotional relationship with users. Windows also states that sound can be used as a supplement to the visual UI (User Interface). For example, adding sound effects to notifications makes them more easily noticed, especially if the user is not viewing the screen when an event occurs.

\section{RELATED WORK}

\subsection{Feeling of Notification Message Color}

Sillic (2016) conducted a study on how the color of a warning banner message affects a user's decision-making process. They employed different colored warning banner messages: black, blue, yellow, red, green, and white. They conducted their experiment in two different countries: the United States and India. Yellow, red, and green were more effective warning colors. However, in the United States, red was the most effective warning color, while yellow was the most effective one in India. Thus, users' feelings depend on the cultural environment. Their study investigated how users feel about the notification message color, but did not consider the notification message sound.

\subsection{Information Display by Signaling Sounds}

Wake noted that humans originally used the five senses to collect information, but most information was received visually. It was thought that representing information with sound is an effective alternative to representing information with sight, and that the use of a sine (non-verbal) sounds plays an important role. Hence, Wake (2005) proposed using non-verbal sounds in a SUI (Sound User Interface). Similar to a GUI (Graphical User Interface), which has both functionality and aesthetics, Wake believed that a SUI must also 
satisfy both functionality and aesthetics. However, both characteristics of the current sign sounds have yet to be fully considered. Hence, these characteristics should be studied to improve the design methodology and guidelines from a functional point of view as well as to improve developers' awareness of sign sounds from a design point of view. Wake's study investigated the use of sound to display information, but did not consider the use of sounds according to users' feelings.

\section{SOUND-ASSIGNMENT METHOD}

This chapter describes a method to assign sounds to notification messages based on the importance of the messages and users' feelings.

\subsection{Assignment Flow}

Figure 1 overviews the process of assigning sounds to notification messages. Software developers classify notification messages into levels according to the notification message importance guidelines, which are described in Section 4.2. In addition, software developers should prepare candidate sounds. Then a user listens to the candidate sounds and evaluates them according to a sound evaluation questionnaire. Based on the answers in the questionnaire, sounds are assigned to notification messages by the sound-assignment algorithm of our method.

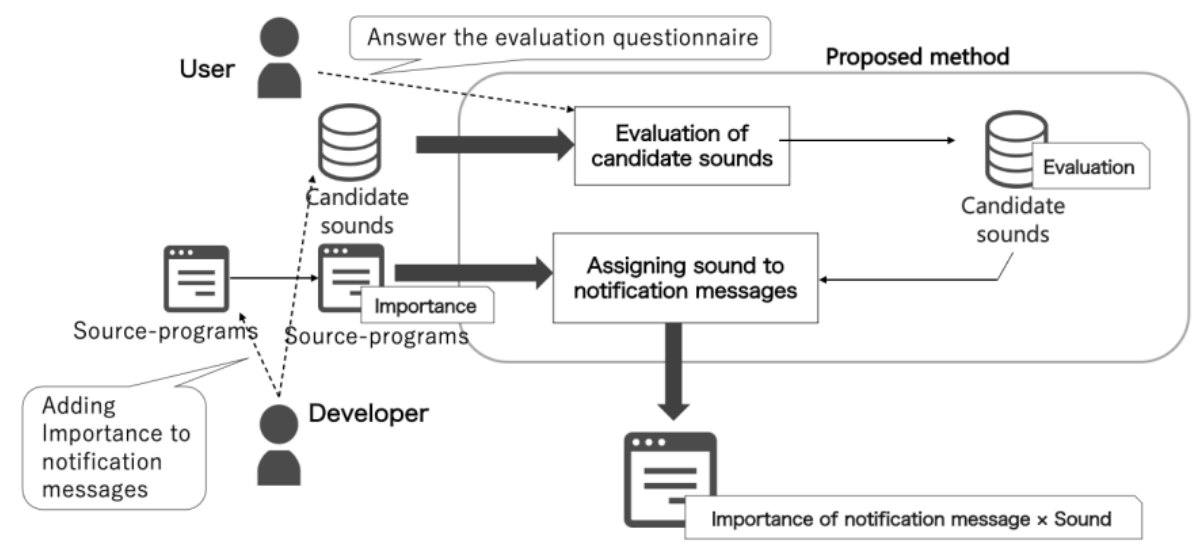

Figure 1. Sound-assignment process for notification messages

\subsection{Guidelines to Determine Importance Levels of Notification Messages}

There are various types of notification messages. Although user interface guidelines often define several types of notification messages, it is defined in terms of software behavior and not necessarily defined according to the importance level recognized by users. In this study, we define guidelines for software developers to determine the importance levels of software notification messages (Table 1). There are four importance levels, where level 1 is least important and level 4 is most important.

Table 1. Guidelines to determine the importance levels of notification messages and their examples

\begin{tabular}{lll}
\hline Importance level & Content & Example \\
\hline level 4 & The user needs to respond immediately or & Purchase confirmation \\
& something may be lost & Permanent deletion of files \\
level 3 & Users do not necessarily have to respond & Low battery notification \\
& immediately, but something may be lost & Password authentication failure \\
level 2 & Some users need to respond & Email notifications \\
& & OS update notifications \\
level 1 & Users do not have to respond & News and other information \\
\hline
\end{tabular}




\subsection{Evaluation of the Importance Felt for Sound}

To assign sounds, the appropriateness of sounds for notification messages according to users must be measured. Thus, a scale is required to measure the sound characteristics. Iwamiya et al. (2009) conducted a rating experiment using adjective pairs to investigate the possibility of assigning chords as sounds to convey messages. Table 2 shows the 10 adjective pairs for the sound impressions. These adjectives were used to evaluate chords and not necessarily the appropriateness of sounds for notification messages. To measure the appropriateness of sounds for notification messages, appropriate adjectives for the scales must be selected and weighted.

Here, we investigate the applicable levels of the adjectives to actual notification sounds. Nine sounds were prepared: Earthquake Early Warning Sound, XP Alert, Windows Error Sound, Windows User Account Control, Windows Low Battery, Windows Notify Calendar, LINE Message Ringtone, Windows Information Bar, and Windows Notify System Generic. We assumed that the earthquake early warning sound, XP warning sound, and Windows error sound are importance level 4, Windows user account control and Windows low battery are level 3, Windows Notify Calendar and LINE message ringtones are level 2, and Windows Notify System Generic and Windows information bar are level 1. Nineteen subjects listened to these sounds and were asked to rate their impressions on a six-point Likert scale, where a higher value indicated a higher importance. For example, for the adjective pair "relaxed/tense", there were 6 levels of evaluation, where 0 means no tension and 5 means a lot of tension.

Table 3 shows the average results of the questionnaire for each sound. Although Table 3 does not include characteristics of each importance level, the earthquake early warning sound has a relatively high value compared to the other sounds. A t-test was conducted to calculate the distance between the sound of earthquake early warning and the other sounds for each of the 10 adjective pairs of sound impressions. A significant difference was set to the commonly used p-value of 0.05 . Table 4 lists the t-values for each adjective. There were 166 degrees of freedom. According to the t-distribution table, the t-value for the 166 degrees of freedom is 1.9744 . Because the values of all 10 adjective pairs significantly differ from those of the earthquake early warning, the sound value of the earthquake early warning is greater than the mean value of the other sounds for all 10 adjective pairs.

As mentioned in Section 2.1, we believe that the earthquake early warning sound is an appropriate reference for high-importance notifications. Because all adjective pairs have high values for the earthquake early warning sound, all 10 adjective pairs of sound impressions can be used as a scale to measure the appropriateness of sounds for notification messages. A higher value indicates a higher appropriateness. In addition, the sound of earthquake early warning must (1) give a feeling of urgency but (2) not give a feeling of uncomfortability or anxiety. Accordingly, it is necessary to exclude uncomfortable sounds. Thus, the "comfortable/uncomfortable" adjective pair was used as a cut-off point to exclude extreme values. The other adjectives were scored and used. In the scoring, we divided the evaluation into three groups: $0-1.67$ out of a maximum value of 5 is the low-scoring group, 1.6-3.33 is the medium scoring group, and 3.33-5 is the high-scoring group.

According to the evaluation of each adjective pair for the earthquake early warning sound in Table 3 , no adjective pair belongs to the low-scoring group, two adjective pairs, "bright/dark" and "light/heavy", belong to the medium-scoring group, and the other seven adjective pairs belong to the high-scoring group. Hence, the higher the score, the more appropriate the sound.

In our method, the sounds are compared. First, the scores of the high-scoring group are compared. If two sounds have the same score using the high-scoring group, the scores of the medium-scoring group are also used.

Table 2. Ten adjective pairs of sound impressions

\begin{tabular}{l}
\hline Adjectives \\
\hline relaxed / tense \\
familiar / unfamiliar \\
comfortable / uncomfortable \\
quiet / noisy \\
bright / dark \\
safe / dangerous \\
slow / busy \\
stable / unstable \\
light / heavy \\
common / unique
\end{tabular}


Table 3. Experimental results of the evaluation for sound adjectives

\begin{tabular}{|c|c|c|c|c|c|c|c|c|c|c|}
\hline & $\begin{array}{l}\text { relaxed } \\
\text { tense }\end{array}$ & $\begin{array}{l}\text { familiar } \\
\text { unfamiliar }\end{array}$ & $\begin{array}{l}\text { comfortable } \\
\text { uncomfortable }\end{array}$ & $\begin{array}{l}\text { quiet } \\
\text { noisy }\end{array}$ & $\begin{array}{l}\text { bright } \\
\text { dark }\end{array}$ & $\begin{array}{l}\text { safe } \\
\text { dangerous }\end{array}$ & $\begin{array}{l}\text { slow } \\
\text { busy }\end{array}$ & $\begin{array}{l}\text { stable } \\
\text { unstable }\end{array}$ & $\begin{array}{l}\text { light } \\
\text { heavy }\end{array}$ & $\begin{array}{l}\text { common } \\
\text { unique }\end{array}$ \\
\hline $\begin{array}{l}\text { Earthquake Early } \\
\text { Warning }\end{array}$ & 4.45 & 4.15 & 4.2 & 3.9 & 3.3 & 4.6 & 4.25 & 4.45 & 2.5 & 4.05 \\
\hline XP Alert & 2.25 & 2.95 & 2.65 & 1.8 & 3.5 & 2.7 & 2.3 & 2.15 & 3.8 & 2.47 \\
\hline Windows Error & 2.11 & 2.16 & 2.32 & 1.79 & 1.11 & 2.16 & 1.95 & 2.39 & 0.84 & 1.84 \\
\hline $\begin{array}{l}\text { Windows User } \\
\text { Account Control }\end{array}$ & 1.47 & 1.74 & 1.63 & 0.95 & 1.89 & 1.79 & 1.21 & 1.95 & 1.26 & 2.37 \\
\hline $\begin{array}{l}\text { Windows Low } \\
\text { Battery }\end{array}$ & 1.68 & 2.16 & 2.32 & 1.58 & 2.21 & 2.0 & 1.63 & 2.16 & 1.47 & 1.84 \\
\hline $\begin{array}{l}\text { Windows Notify } \\
\text { Calendar }\end{array}$ & 0.82 & 1.24 & 1.35 & 1.12 & 1.35 & 1.12 & 1.0 & 1.88 & 1.29 & 2.29 \\
\hline $\begin{array}{l}\text { LINE Message } \\
\text { Ringtone }\end{array}$ & 1.56 & 1.11 & 2.06 & 2.17 & 1.17 & 1.22 & 2.78 & 1.5 & 0.72 & 2.56 \\
\hline $\begin{array}{l}\text { Windows } \\
\text { Information Bar }\end{array}$ & 2.67 & 3.28 & 3.17 & 2.78 & 1.83 & 2.78 & 2.67 & 2.78 & 1.67 & 2.89 \\
\hline $\begin{array}{l}\text { Windows Notify } \\
\text { System Generic }\end{array}$ & 0.94 & 1.56 & 1.78 & 1.17 & 1.78 & 1.39 & 1.06 & 1.5 & 1.5 & 2.83 \\
\hline
\end{tabular}

Table 4. T-values for each of the other sound adjectives compared with the sound of earthquake early warning

\begin{tabular}{lllllllllll}
\hline & $\begin{array}{l}\text { relaxed } \\
\text { tense }\end{array}$ & $\begin{array}{l}\text { familiar } \\
\text { unfamiliar }\end{array}$ & $\begin{array}{l}\text { comfortable } \\
\text { uncomfortable }\end{array}$ & $\begin{array}{l}\text { quiet } \\
\text { noisy }\end{array}$ & $\begin{array}{l}\text { bright } \\
\text { dark }\end{array}$ & $\begin{array}{l}\text { safe } \\
\text { dangerous }\end{array}$ & $\begin{array}{l}\text { slow } \\
\text { busy }\end{array}$ & $\begin{array}{l}\text { stable } \\
\text { unstable }\end{array}$ & $\begin{array}{l}\text { light } \\
\text { heavy }\end{array}$ & $\begin{array}{l}\text { common } \\
\text { unique }\end{array}$ \\
\hline t-value & 13.96 & 6.76 & 8.96 & 9.64 & 4.08 & 18.85 & 8.72 & 12.92 & 2.72 & 6.66 \\
\hline
\end{tabular}

\subsection{Appropriateness Evaluation of Sounds}

In our method, sounds are assigned to notification messages according to the following algorithm:

(1) Users listen to candidate sounds prepared by the software developer and rate each sound on a 6-point Likert scale from 0 to 5 for the 10 adjective pairs of sound impressions shown in Table 2. As explained in Section 4.3, a higher score indicates a higher appropriateness. Table 5 shows an example of an evaluation.

(2) For each sound, the scores are calculated according to Formula (i), using the ratings of each adjective pair for the high-scoring group ("relaxed/tense", "familiar/unfamiliar", "quiet/noisy", "safe/dangerous", "slow/busy", "stable/unstable", and "common/unique") and medium-scoring group ("bright/dark" and "light/heavy") defined in Section 4.3. In this formula, $n$ is the number of adjectives in a group, $v$ is the user rating for each adjective, and $t$ is the $\mathrm{t}$-value for each adjective shown in Table 4. Table 6 shows an example of the results.

$$
\text { score }=\sum_{i=1}^{n} v_{i} t_{i}
$$

(3) Sounds that are very uncomfortable are excluded (i.e., those that have a "comfortable/uncomfortable" adjective value of 5 among the candidate sounds). As an example, Sound 4 in Table 5 is excluded due to this criterion.

(4) Based on the scores of the high-scoring group, the remaining sounds in step (3) are ranked in descending order by score. If sounds have the same score, then the scores of the medium-scoring group are evaluated. If the scores in the medium-scoring group are the same, then the sounds are assigned the same rank. Table 6 shows an example ranking.

(5) The remaining $k$ sounds from step (3) are divided into four groups of $k / 4$ sounds in order of rank in step (4). Table 7 shows an example. The highest-ranked sound in the highest-ranked group is assigned to a notification message of importance level 4, as shown in Table 1. For the second group, the sound of the middle rank within the group is assigned to importance level 3. For the third group, the sound of the middle rank within the group is assigned to importance level 2. Finally, the lowest-ranked sound within the lowest-ranked group is assigned to importance level 1. Table 7 shows an example of the assignment results, where a circle indicates an assigned sound. 
Table 5. Example of each sound evaluation by the number of users

\begin{tabular}{lllllllllll}
\hline & $\begin{array}{l}\text { relaxed } \\
\text { tense }\end{array}$ & $\begin{array}{l}\text { familiar } \\
\text { unfamiliar }\end{array}$ & $\begin{array}{l}\text { comfortable } \\
\text { uncomfortable }\end{array}$ & $\begin{array}{l}\text { quiet } \\
\text { noisy }\end{array}$ & $\begin{array}{l}\text { bright } \\
\text { dark }\end{array}$ & $\begin{array}{l}\text { safe } \\
\text { dangerous }\end{array}$ & $\begin{array}{l}\text { slow } \\
\text { busy }\end{array}$ & $\begin{array}{l}\text { stable } \\
\text { unstable }\end{array}$ & $\begin{array}{l}\text { light } \\
\text { heavy }\end{array}$ & $\begin{array}{l}\text { common } \\
\text { unique }\end{array}$ \\
\hline Sound 1 & 2 & 3 & 3 & 3 & 3 & 4 & 2 & 4 & 2 & 2 \\
Sound 2 & 2 & 4 & 4 & 4 & 3 & 3 & 3 & 3 & 3 & 2 \\
Sound 3 & 2 & 2 & 1 & 3 & 1 & 2 & 1 & 1 & 1 & 2 \\
Sound 4 & 4 & 5 & 5 & 5 & 2 & 3 & 3 & 4 & 2 & 2 \\
Sound 5 & 0 & 4 & 3 & 3 & 2 & 2 & 2 & 2 & 2 & 3 \\
Sound 6 & 3 & 2 & 2 & 2 & 3 & 2 & 2 & 2 & 3 & 3 \\
Sound 7 & 1 & 1 & 1 & 3 & 0 & 2 & 1 & 1 & 0 & 0 \\
Sound 8 & 3 & 2 & 2 & 4 & 1 & 3 & 3 & 2 & 1 & 1 \\
Sound 9 & 2 & 3 & 3 & 3 & 1 & 3 & 3 & 3 & 2 & 4 \\
Sound 10 & 2 & 3 & 3 & 3 & 1 & 3 & 3 & 3 & 2 & 4 \\
\hline
\end{tabular}

Table 6. Example of the high- and medium-scoring groups' scores, their rankings, and assigned importance levels

\begin{tabular}{lllll}
\hline & $\begin{array}{l}\text { High-scoring } \\
\text { group scores }\end{array}$ & $\begin{array}{l}\text { Medium- } \\
\text { scoring group } \\
\text { scores }\end{array}$ & Rank & $\begin{array}{l}\text { Assigned } \\
\text { importance level }\end{array}$ \\
\hline Sound 1 & 235.31 & 17.71 & 2 & - \\
Sound 2 & 241.94 & 20.44 & 1 & 4 \\
Sound 3 & 143.16 & 6.81 & 7 & - \\
Sound 4 & 292.60 & 13.62 & - & - \\
Sound 5 & 163.80 & 13.62 & 6 & 2 \\
Sound 6 & 175.86 & 20.44 & 5 & - \\
Sound 7 & 109.09 & 0 & 8 & - \\
Sound 8 & 209.39 & 6.81 & 4 & 3 \\
Sound 9 & 106.12 & 6.81 & 9 & 1 \\
Sound 10 & 225.53 & 9.54 & 3 & - \\
\hline
\end{tabular}

Table 7. Example groupings of candidate sounds by importance level

\begin{tabular}{ll}
\hline Importance level & Grouped candidate sounds \\
\hline 4 & OSound 2, Sound 1, Sound 10 \\
3 & OSound 8, Sound 6 \\
2 & OSound 5, Sound 3 \\
1 & Sound 7, OSound 9 \\
\hline
\end{tabular}

\section{EXPERIMENT}

To investigate the effectiveness of the proposed sound-assignment method, we conducted an experiment involving 20 subjects.

\subsection{Experimental Design}

The 20 subjects listened to the 10 candidate sounds and rated each one (Table 2). The candidate sounds were free materials (Kurage-kosho, 2014). These sounds were selected based on the intention according to the authors. The intentions were written as tags and comments such as "sounds that can be used as error sounds" and "bright images". We assumed that these sounds were appropriate for importance levels 1 to 4 of notification messages. For each sound, the subjects selected which level of importance they thought was appropriate for the notification message (Table 1). However, if a subject felt that a sound was inappropriate for any level, they selected none and provided their reason. 


\subsection{Experimental Results}

To determine the sounds assigned to the four importance levels of notification messages for each subject, the sound-assignment algorithm in Section 4.4 was applied to the subject's answers. Table 8 shows subjects' answers of the appropriate levels for the assigned sounds by importance level of the notification message. For example, when Sound 1 was assigned to importance level 1 of notification message, but a subject thought the sound was appropriate for importance level 2, Table 8 shows a "2" in the cell of importance level 1 by subject. Then the gap between the importance level of the assigned sound and the importance level of the notification message that the subject thought was appropriate for the sound was calculated. For example, Subject 1 felt that the sound assigned to importance level 3 should be assigned to level 4, the gap is $|4-3|=1$. Table 9 shows the total number of gaps and the average of the gaps for all subjects for each importance level. However, here "other" indicates the case when a subject responded that the sound was inappropriate for all importance levels. One reason for the lack of appropriate importance level was that a long sound was perceived as an OS boot sound rather than a notification sound. Additionally, all subjects selected either importance level 1 or importance level 2 as appropriate for any of the 10 candidate sounds, but some subjects did not identify any sound as appropriate for importance level 3 or 4.

Table 8. Importance assignment sound levels selected by subject

\begin{tabular}{lllll}
\hline Subject & Importance level 4 & Importance level 3 & Importance level 2 & Importance level 1 \\
\hline 1 & 3 & 3 & 1 & 1 \\
2 & 3 & 2 & N/A & 1 \\
3 & 2 & 3 & 1 & 1 \\
4 & 2 & 2 & 2 & 1 \\
5 & 2 & 1 & 1 & 1 \\
6 & 3 & 2 & 1 & 2 \\
7 & 3 & 3 & 3 & 1 \\
8 & 2 & 4 & 1 & 1 \\
9 & 3 & 2 & 1 & 1 \\
10 & 3 & 1 & 1 & 1 \\
11 & 4 & 2 & 1 & 1 \\
12 & 3 & 1 & 2 & 1 \\
13 & 3 & 3 & 1 & 1 \\
14 & 2 & 1 & N/A & 1 \\
15 & 3 & 1 & 1 & 1 \\
16 & 3 & 2 & 1 & 1 \\
17 & 3 & 1 & 1 & 1 \\
18 & 4 & 1 & 1 & 1 \\
19 & 3 & 2 & 3 & 1 \\
20 & 2 & 2 & 1 & 1 \\
\hline
\end{tabular}

Table 9. Gap of importance levels for notification messages between the assignment by our method and users' feelings to the sounds

\begin{tabular}{ll}
\hline Gap & Total number \\
\hline 0 & 30 \\
1 & 35 \\
2 & 13 \\
3 & 0 \\
4 & 0 \\
other & 2 \\
average & 0.7625 \\
\hline
\end{tabular}

\subsection{Discussion}

The average gap of the importance levels for notification messages between the assignment by our method and users' feelings to the sounds was 0.7625 , which was less than 1 (Table 9). In the cases with a gap of 2, in 7 of the 13 cases, the subjects did not select level 3 or level 4 as an importance level appropriate for the 
candidate sounds. In all but one of the remaining six cases, although the subject selected level 3 or higher for candidate sounds, these sounds were omitted in step (3) of our sound-assignment algorithm in Section 4.4. Hence, the average gap was small and in the case where the gap was large, the candidate sound did not match the subjects' feelings. Overall, the sound-assignment method described in Section 4 is considered to be effective.

\section{CONCLUSION}

Herein we propose a method to assign notification sounds to notification messages according to the user's individual feelings. Our method allows users to notice and take appropriate actions for important notification messages.

Future works include to:

1) build a support system to develop software with an assignment function of the notification sound based on our method, and

2) confirm the appropriateness of users' actions based on the assigned notification sounds by our method.

\section{REFERENCES}

Ifukube, A, 2013, The Birth Story of the Earthquake Early Warning Chime. JAS Journal, Vol. 53, No. 2, pp.4-10.

Iwamiya, S. et al, 2009. On the Effectiveness of Using Musical Chords for Auditory Signals. The Japanese Journal of Ergonomics, Vol. 45, No. 6, pp. 329-335.

Japan Meteorological Agency, 2007. What is an Earthquake Early Waring? Available at: https://www.jma.go.jp/jma/en/Activities/eew1.html [Accessed 3 Jan. 2021].

Kurage-kosho, 2014. System Sound and Electronic Sound. Available at: http://www.kurage-kosho.info/system.html [Accessed 3 Jan. 2021].

MacOS Human Interface Guidelines, 2021. Notifications. Available at: https://developer.apple.com/design/humaninterface-guidelines/macos/system-capabilities/notifications/ [Accessed 3 Jan. 2021].

Microsoft, 2020. Sound. Available at: https://docs.microsoft.com/en-us/windows/win32/uxguide/vis-sound [Accessed 3 Jan. 2021].

Sakai, M. et al, 2019. Assigning sounds according to the importance of notification messages and users' feelings. The 81 st National Convention Lecture and Paper Collection, Vol. 2019, No. 1, pp. 257-258 (in Japanese).

Sillic, M. et al, 2016. Understanding Colour Impact on Warning Messages: Evidence from US and India. Proceedings of the 2016 CHI Conference Extended Abstracts on Human Factors in Computing Systems. San Jose, USA, pp. 2954-2960.

Wake, S, SUI(Sound User Interface) : Auditory Information Display used Sign Sound and Its Design. Proceedings of the Human Interface Symposium. Fujisawa, Japan, pp. 105-110. 